EPJ Web of Conferences 47, 09002 (2013)

DOI: $10.1051 /$ epjconf/20134709002

(C) Owned by the authors, published by EDP Sciences, 2013

\title{
Realistic limitations of detecting planets around young active stars
}

\author{
S.V. Jeffers ${ }^{1, a}$, J.R. Barnes ${ }^{2}$, H. Jones ${ }^{2}$, D. Pinfield ${ }^{2}$ \\ and RoPACS collaboration \\ ${ }^{1}$ Institut fur Astrophysik, Georg-August Universitat Goettingen, Friedrich-Hund-Platz 1, \\ 37077 Goettingen, Germany \\ ${ }^{2}$ Centre for Astrophysics Research, University of Hertfordshire, College Lane, Hatfield, Herts \\ AL10 9AB, UK
}

\begin{abstract}
Current planet hunting methods using the radial velocity method are limited to observing middleaged main-sequence stars where the signatures of stellar activity are much less than on young stars that have just arrived on the main-sequence. In this work we apply our knowledge from the surface imaging of these young stars to place realistic limitations on the possibility of detecting orbiting planets. In general we find that the magnitude of the stellar jitter is directly proportional to the stellar vsini. For $\mathrm{G}$ and $\mathrm{K}$ dwarfs, we find that it is possible, for models with high stellar activity and low stellar vsini, to be able to detect a $1 \mathrm{M}_{\text {Jupiter }}$ mass planet within 50 epochs of observations and for the $\mathrm{M}$ dwarfs it is possible to detect a habitable zone Earth-like planet in 10s of observational epochs.
\end{abstract}

\section{INTRODUCTION}

The vast majority of the currently known 900 extra-solar planets have been detected using the radial velocity method. The large surveys that use this method are generally aimed at detecting planets around older and less active stars. However to fully understand how such planetary systems evolve it is necessary to also observe and detect planets around young active stars.

One major obstacle in detecting planets around young (zero age main-sequence) stars is that these stars are generally more active than older stars such as the Sun. Activity signatures in the form of starspots, plage and convective motions can distort absorption line profiles leading to time variant line asymmetries. Such distortions can result in biased RV measurements that can either mask a planet's RV signature and/or give a spurious detection. In addition to the degree of stellar activity how the activity signatures are distributed on the stellar surface is also important. Lower latitude spots, such as those observed on the Sun, result in a higher RV jitter than high latitude spots such as those observed on young active Solar-type stars [1]. In this work we place realistic limitations on the possibilities for detecting planets around young active $\mathrm{G}, \mathrm{K}$, with thin convective zones, and M dwarfs, which are almost fully convective.

\section{STELLAR ACTIVITY ON G, K AND M DWARFS}

In this Section we give an overview of the known signatures of stellar activity on $\mathrm{G}, \mathrm{K}$ and $\mathrm{M}$ dwarfs.

ae-mail: jeffers@astro.uni-goettingen.de

This is an Open Access article distributed under the terms of the Creative Commons Attribution License 2.0, which permits unrestricted use, distribution, and reproduction in any medium, provided the original work is properly cited. 


\section{EPJ Web of Conferences}

\section{$2.1 \mathrm{G}$ and $\mathrm{K}$ dwarfs}

\subsection{Starspots}

Doppler Images of $\mathrm{G}$ and $\mathrm{K}$ dwarfs typically show polar and high latitude starspot coverage, with some low latitude spot coverage. In this analysis we use Doppler Images of the G dwarf HD 141943 and the $\mathrm{K}$ dwarf $\mathrm{AB}$ Dor as realistic input spot distributions.

\subsection{Plage}

The distribution and coverage of plage on active stars is not well understood. However, global constraints can be obtained from the simultaneous monitoring of photometric brightness and $\mathrm{Ca} H \& \mathrm{~K}$ such as described by [8]. They find that for younger, and consequently more active stars, become fainter as their $\mathrm{Ca} H \& \mathrm{~K}$ emission increases in contrast to the Sun [9].

\subsection{Unresolved starspots}

Tomographic Images of young active stars show starspot patterns that are significantly different to that observed on the Sun, with high latitude spots on $\mathrm{G}$ and $\mathrm{K}$ dwarfs and a uniform spot coverage on early $\mathrm{M}$ dwarfs. For all spectral types, the typical spot coverage fraction reconstructed using Doppler Imaging techniques is approximately $10 \%$. However, other independent analysis such as that using $\mathrm{TiO}$ band analysis indicates that starspot coverage could be at high as 20 to $40 \%$ [7]. This discrepancy could be resolved if the stars have many small starspots that are below the resolution limits of Doppler Imaging. To understand how this extra contribution could impact the detection of planets we add a spot coverage of $29.5 \%$ following the method of [5].

\subsection{Stellar activity models: $G$ and $K$ dwarfs}

The final modelled activity distributions are modelled on an immaculate $\mathrm{G}$ and $\mathrm{K}$ dwarf star

1. Spots only - modelled from the tomographic Doppler images of HD 141943 and AB Dor

2. Spots + Plage - as for case 1 . but with the addition of large regions of plage surrounding starspots

3. Spots + Plage + Random Spots - case 2. with many small random (spot + ) plage areas to mimic Solar plage distributions. these stars are spot dominated rather than plage dominated as in the case of the Sun

4. Immaculate Star: as above, but without any stellar activity.

\subsection{Stellar activity models: M dwarfs}

In contrast to the $\mathrm{G}$ and $\mathrm{K}$ dwarfs, Doppler Images of $\mathrm{M}$ dwarfs show uniform spot coverage, with spots at low and high latitudes, and without a polar cap. The spot coverage fraction is similar with $10 \%$ coverage [2]. Similarly as for the $\mathrm{G}$ and $\mathrm{K}$ dwarfs $\mathrm{TiO}$ band analysis indicates a much higher spot coverage of 20 to $40 \%$ [7].

To understand how the degree of spot coverage impacts the detections of planets on M dwarfs, we model a random distribution of spots (following the method of [5], with a spot coverage levels of (1) Solar minimum (2) Solar maximum (3) $1.89 \%$ (4) $8.95 \%$ (5) $29.5 \%$ and (6) $62.4 \%$.

\subsection{Generation of line profiles}

Line profiles are then generated for these stellar activity models using the stellar imaging code DoTs [3]. DoTs can model both cool dark spots and regions of hot bright plage using a 3D stellar model. Further details are given in [4] and [1]. 


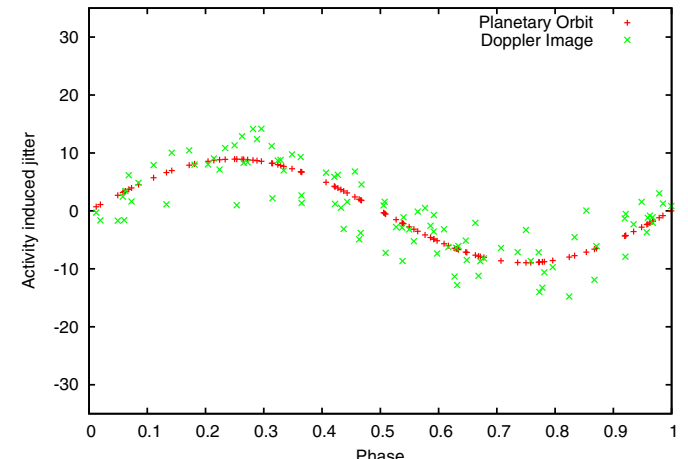

(a) Doppler Image

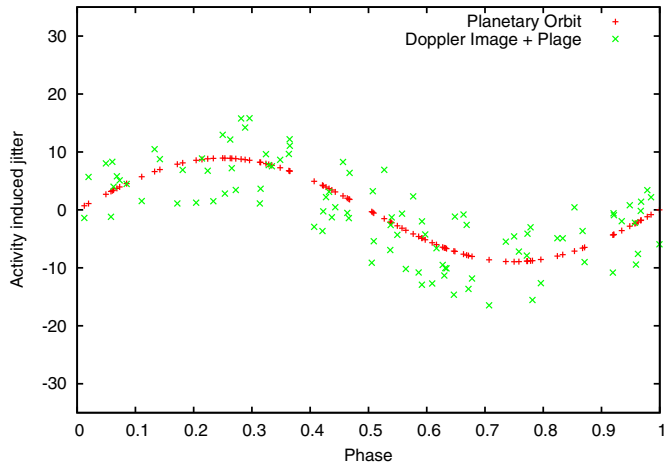

(b) Doppler Image + Plage

Figure 1. The impact of stellar activity on the simulated radial velocity profile, with the planetary radial velocity curve plotted in red and the stellar activity jitter plotted as green points. The models shown here are for a planet mass $=10 \mathrm{M}_{\text {earth }}$ orbiting $\mathrm{G}$ dwarf at $0.01 \mathrm{AU}$. The stellar activity originates from Doppler Image (left), Doppler image and plage (right)

\section{MODELLING RADIAL VELOCITY DATA}

A star's radial velocity profile is comprises (1) jitter caused by activity on the host star (2) the radial velocity induced by a planet orbiting its host star and also (3) the achievable instrumental precision.

\subsection{Radial velocities induced by activity jitter}

Star with an axial inclination $=90^{\circ}$ are simulated with 90 line profiles per rotational phase and a vsini $=1,2,5,10,20,50,100 \mathrm{~km} \mathrm{~s}^{-1}$ and a spectral resolution $=70,000$. The radial velocity profiles for the model of Doppler Image, and Doppler Image + Plage are plotted in Fig. 1. The addition of plage is shown to slightly increases the RMS jitter. In agreement with [1] their is a direct correlation between induced activity jitter and the stellar vsini.

\subsection{Radial velocities induced by planets}

\subsubsection{G and $K d$ warfs}

For $\mathrm{G}\left(\mathrm{M}_{\text {star }}=1.0\right)$ and $\mathrm{K}$ dwarfs $\left(\mathrm{M}_{\text {star }}=0.8\right)$, the planetary mass is set to be equal to $1,2,5,10,20$ $\mathrm{M}_{\text {Earth }}$ and 1, 2, $5 \mathrm{M}_{\text {Jupiter }}$ orbiting at heliocentric distances of 0.01, 0.02, 0.05, 0.1, 0.2, 0.5, $1 \mathrm{AU}$.

\subsubsection{Mdwarfs}

For the M dwarfs low-mass planets are modelled orbiting centre of the host star's habitable zone with planetary masses $=\mathbf{M}_{\text {planet }}=1,2,5,10$ and $20 \mathbf{M}_{\text {earth }}$ and $\mathbf{M}_{\text {star }}=0.1,0.2$ and $0.5 \mathrm{M}_{\text {sun }}$.

\subsection{Instrumental precision}

The achievable instrumental precision is computed for G, K and M dwarfs using a Signal-to-Noise ratio of 100. This is described in more detail by Jeffers et al. (2013) for $G$ and $K$ dwarfs and Barnes et al. (2011) for M dwarfs. For all spectral types, the achievable precision is a function of stellar vsini. 


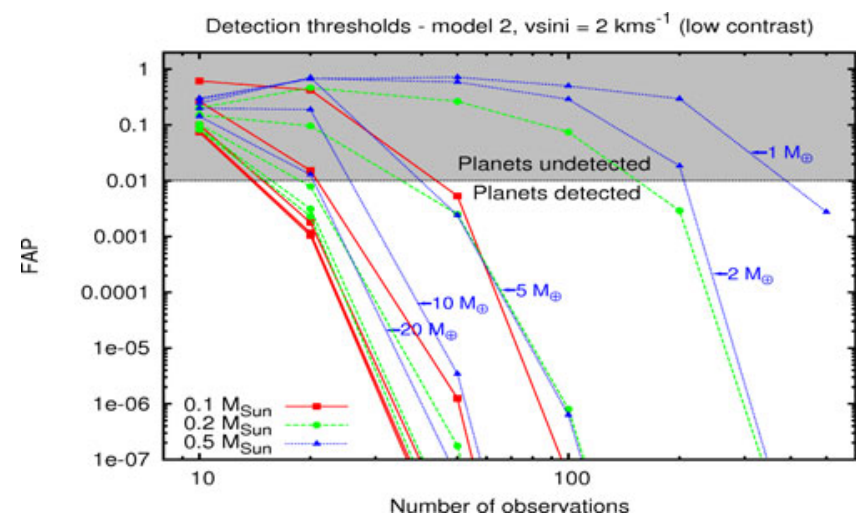

Figure 2. M dwarfs: detection false alarm probabilities (FAPs) as a function of number of observations epochs for $1,2,5,10$ and $20 \mathrm{M}_{\text {earth }}$ planet orbiting at in the habitable zone of a 0.1, 0.2, and $0.5 \mathrm{M}_{\text {sun }} \mathrm{M}$ dwarf [red, green and blue lines in plots]. The stellar activity model used for these plots is the solar maximum model. The horizontal line indicates the 1 per cent false alarm probability $(\mathrm{FAP}=0.01)$ with the region above this line representing undetected planets. All points with FAP $<0.01$ are considered as planet detections.
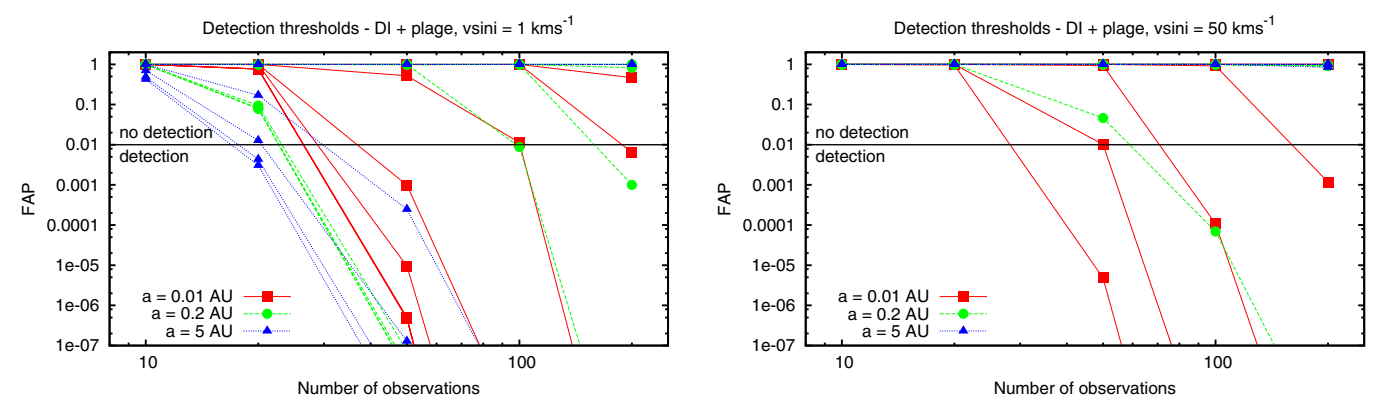

Figure 3. G dwarfs: detection false alarm probabilities (FAPs) as a function of number of observations epochs for $1,2,5,10,20,159,318,636$ and $1589 \mathrm{M}_{\text {earth }}$ planet orbiting at $0.01 \mathrm{AU}, 0.2 \mathrm{AU}$ and $5 \mathrm{AU}$ [red, green and blue lines in plots]. The stellar activity model used for these plots is the model containing Doppler Image and additional plage. The horizontal line indicates the 1 per cent false alarm probability $(\mathrm{FAP}=0.01)$ with the region above this line representing undetected planets. All points with FAP $<0.01$ are considered as planet detections. The plot on the left is for a vsini $=1 \mathrm{~km} \mathrm{~s}^{-1}$ and vsini $=50 \%$ on the right.

\subsection{Simulating the detection of planets around active dwarfs}

For each for the above star-planet combinations we generate radial velocity profiles for a range of observations epochs corresponding to $10,20,50,100 \& 200$ epochs of observation on consecutive nights with a random observational phase. For each planet model, stellar activity and instrumental precision jitter is added to the radial velocity profile.

\section{PLANET DETECTION}

To determine realistic limitations of detecting planets around young active $\mathrm{G}, \mathrm{K}$ and $\mathrm{M}$ dwarfs we apply a Lomb Scargle periodogram analysis to the simulated radial velocity profiles that have added jitter from stellar activity and instrumental precision. The results of this analysis are plotted in Fig. 2, where the false-alarm probability is plotted against the total number of observations far a range of vsini values. 


\section{Hot Planets and Cool Stars}

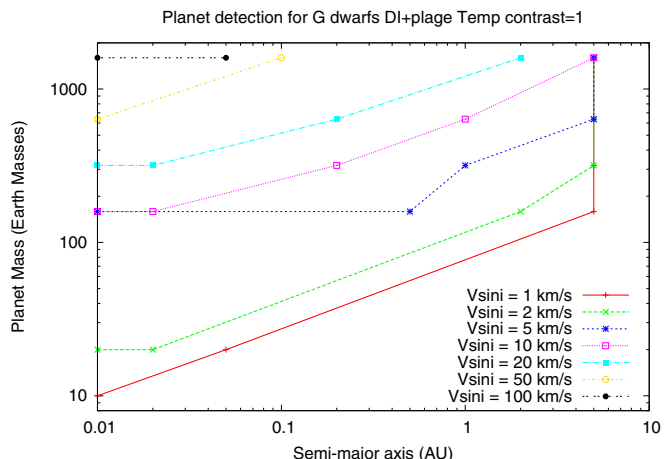

(a) $\mathrm{G}$ dwarfs temp contrast $=1$

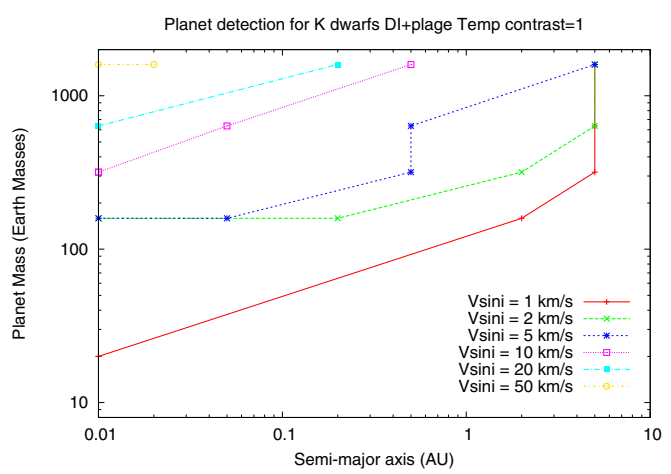

(c) $\mathrm{K}$ dwarfs temp contrast $=1$

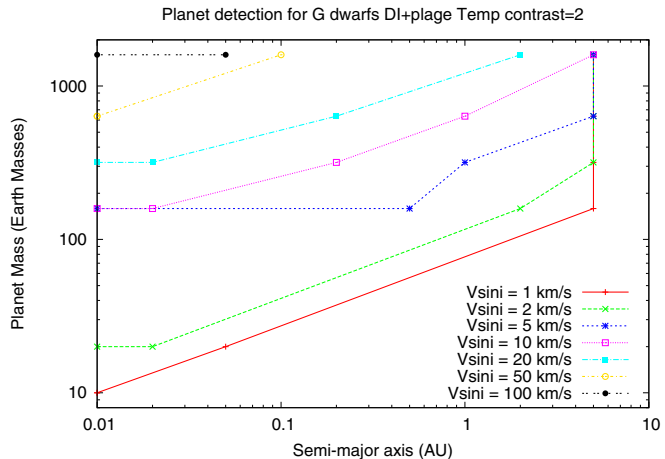

(b) G dwarfs temp contrast $=2$

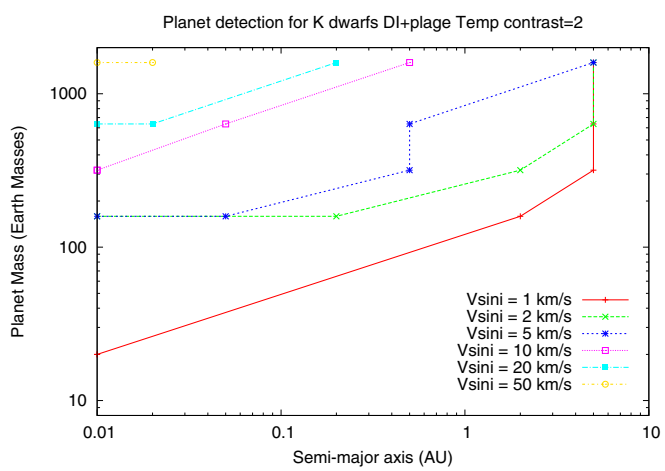

(d) $\mathrm{K}$ dwarfs temp contrast $=2$

Figure 4. Planet detection as a function of planetary mass and star-planet separation. Each point is the lowest mass planet detected within 50 observational epochs in Figure 3 for a given star planet separation. Also shown are the two photosphere/spot temperature contrast ratio. The left panel corresponds to a contrast ratio of $\mathrm{T}_{\text {spot } 1}=0.65 \mathrm{~T}_{\text {phot }}$ and right panels for a ratio of $\mathrm{T}_{\text {spot } 2}=\mathrm{T}_{\text {phot }}-1500 \mathrm{~K}$ for each spectral type.

\subsection{G \& K dwarfs}

The full set of models (not all shown here) shows that the ability to detect a planetary signal clearly decreases with increasing stellar activity and vsini. To summarise, it is possible to detect $1 \mathrm{M}_{\text {Jupiter }}$ planets around active $\mathrm{G}$ and $\mathrm{K}$ dwarfs with up to moderate vsini values. For $\mathrm{G}$ and $\mathrm{K}$ dwarfs we find that temperature contrast is not an important parameter. A summary of these results is plotted in Fig. 4 for 50 observational epochs, where the plotted lines represent lower limits to the detection of planets i.e. all values to the left of the plotted line are planet detections and to the right non-detections. For comparison, the planet detection rates for 100 and 200 observational epochs are shown in Fig. 5.

\subsection{M dwarfs}

As with the $\mathrm{G}$ and $\mathrm{K}$ dwarfs, the ability to detect a planetary signal also clearly decreases with increasing stellar activity and vsini. In summary, with only several $10 \mathrm{~s}$ of epochs it is possible to detect habitable zone Earth-like planets orbiting late $\mathrm{M}$ dwarfs, though this depends strongly on the photosphere/spot temperature contrast ratio. These results are summarised in Fig. 6. 


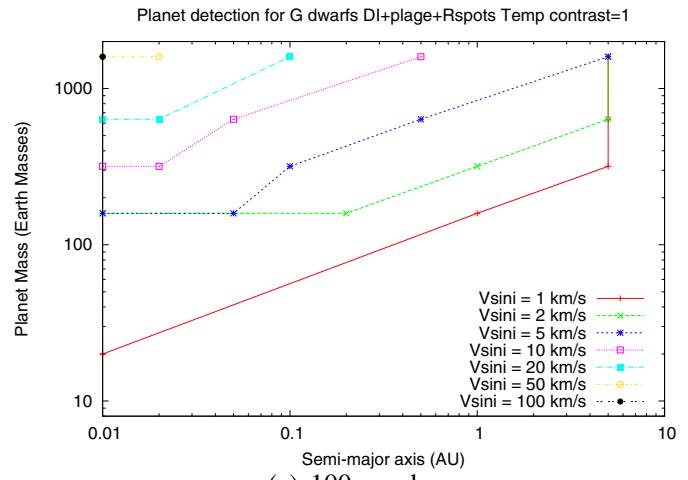

(a) 100 epochs

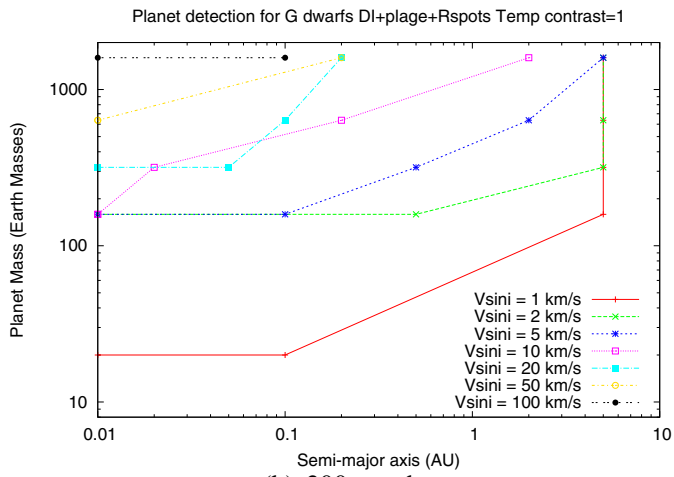

(b) 200 epochs

Figure 5. As for Figure 4 but for (left) 100 observational epochs and (right 200 observational epochs).

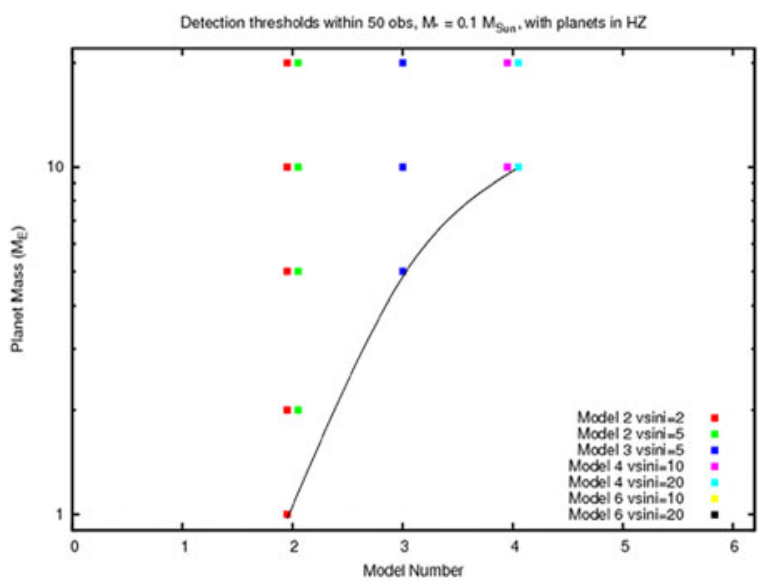

Figure 6. Planet detections as a function of stellar activity (in the form of many random starspots) for M dwarfs. Points to the left of the line represent planet detections while points to the right are non-detections.

\subsection{Future work}

The next steps in this project are to use the same spot patterns for $\mathrm{G}$ and $\mathrm{K}$ dwarfs to make a direct comparison of the effects of stellar mass. On a longer term we will improve planet-hunting techniques and investigate activity removal methods.

\section{References}

[1] Barnes J. R., Jeffers S. V., Jones H. R. A., 2011, MNRAS, 412, 1599

[2] Barnes J. R., James D. J., Collier Cameron A., 2004, MNRAS, 352, 589

[3] Collier Cameron A., 1997, MNRAS, 287, 556

[4] Jeffers S. V., Barnes J. R., Jones H., Pinfield D., submitted

[5] Jeffers S. V.,2005, MNRAS, 359, 729

[6] Meunier N., Desort M., Lagrange A.-M., 2010, A\&A, 512, A39

[7] O’Neal D., Neff J. E., Saar S. H., Cuntz M., 2004, AJ, 128, 1802

[8] Radick R. R., Lockwood G. W., Skiff B. A., Baliunas S. L., 1998, ApJS, 118, 239

[9] Unruh Y. C., Solanki S. K., Fligge M., 1999, A\&A, 345, 635 